\title{
Corrigendum
}

\section{Loss of blood group A antigen expression in bladder cancer caused by allelic loss and/or methylation of the $A B O$ gene}

Yoshitomo Chihara ${ }^{1,2}$, Kokichi Sugano ${ }^{1}$, Ayumi Kobayashi ${ }^{1}$, Yae Kanai ${ }^{3}$, Hidenobu Yamamoto $^{4}$, Masaaki Nakazono ${ }^{4}$, Hiroyuki Fujimoto ${ }^{5}$, Tadao Kakizoe ${ }^{5}$, Kiyohide Fujimoto ${ }^{2}$, Setsuo Hirohashi ${ }^{3}$ and Yoshihiko Hirao ${ }^{2}$

${ }^{1}$ Oncogene Research Unit/Cancer Prevention Unit, Tochigi Cancer Center Research Institute, Tochigi, Japan; ${ }^{2}$ Department of Urology, Nara Medical University, Nara, Japan; ${ }^{3}$ Pathology Division, National Cancer Center Research Institute, Tokyo, Japan; ${ }^{4}$ Department of Urology, Tochigi Cancer Center Hospital, Tochigi, Japan and ${ }^{5}$ Department of Urology, National Cancer Center Hospital, Tokyo, Japan

Laboratory Investigation (2005) 85, 1051. doi:10.1038/labinvest.3700317

Correction to: Laboratory Investigation (2005) 85, 895-907. doi:10.1038/sj.labinvest.3700268

Following the publication of the above paper, the author has identified an error in the affiliation of
Dr Setsuo Hirohashi. The correct affiliation for Dr Hirohashi is shown above. 